\title{
Children's perception of parental attitudes: Russia-Kyrgyzstan cross-cultural study
}

\author{
Tatiana Tikhomirova ${ }^{1,2^{*}}$ and Sergey Malykh ${ }^{1,2}$ \\ ${ }^{1}$ Lomonosov Moscow State University, Department of Psychology, 119991 Moscow, Russia \\ ${ }^{2}$ Psychological Institute of Russian Academy of Education, 125009 Moscow, Russia
}

\begin{abstract}
The article presents the results of a cross-cultural analysis of the perception of parental attitude by children and adolescents. We focused on the indicators of maternal and paternal attitude such as Acceptance, Positive Involvement, Rejection and Hostile Detachment. The Russian sample includes 501 primary school students aged 6.8 to 11.7 years ( $49.9 \%$ boys) and 438 adolescents aged 10.8 to 16.8 years ( $47.1 \%$ boys). The Kyrgyz sample includes 510 primary school students aged from 6.6 to 11.8 years ( $48.5 \%$ boys) and 513 adolescents aged 10.3 to 16.8 years (46.3\% boys). Regardless of the macro environment, schoolchildren perceive the attitude of both parents to a greater extent as emotionally accepting and to a lesser extent as rejecting. At the same time, it is shown that cross-cultural differences in the perception of maternal attitude are manifested only in younger schoolchildren: Kyrgyz children assess maternal attitude as more emotionally accepting with positive involvement and less rejecting. Cross-cultural differences in the perception of paternal attitudes are manifested both in younger schoolchildren and in adolescents: Kyrgyz children assess paternal attitude more positively than Russian peers. The results are interpreted in the context of specific socio-economic conditions.
\end{abstract}

\section{Introduction}

Research of parental attitudes to a child is, as a rule, considered in the context of its influence on individual differences in the psychological characteristics of children of different ages [1-5]. A parental attitude is understood as a set of situationally nonspecific ways of the emotional attitude and behavior of a parent towards a child. According to the psychological and pedagogical literature, two aspects reflecting the parental attitude to a child might be singled out: (1) the degree of emotional acceptance of a child and (2) the kind of involvement in a child's activity $[1,6]$.

It should be noted that maternal attitude is analysed more often. At the same time, it is reported that mothers and fathers build a relationship with their child, participate in child's activities and show their feelings in different ways $[1,7,8$, etc.]. In particular, it was shown that the differences in the educational impact of mothers and fathers are related to the degree of involvement in child's activities, satisfaction of needs and desires of the child and stimulation of the information flow [9, 10].

The aspects of parental attitude can be observed both from the parental position and from the position of a child. A number of studies of family environment highlight a low degree of agreement between parents and children responding to the same psychodiagnostic test: correlation coefficients varied from 0.11 to 0.41 for younger adolescents [11] and from 0.31 to 0.41 for young adults [12]. Such differences in results may be explained by unique individual experience through the prism of which the parental attitude is perceived $[1,7$, $10]$.

A number of studies have shown that child-parent relationship were an important link in the relationship between the socio-economic position of the family and the success of the child $[13,14]$. Moreover, crosscultural differences were reported across a range of aspects of child-parent relationships [15]. For example, cross-cultural studies of the perceptions of the relationship between fathers and mothers were studied in the USA and China, as well as on samples of American families of European and Asian origin (for example, [3, $16,17])$. It was shown that the strict father model is characteristic for American families of Chinese origin but not for families of European origin [18].

It is reported that there are parental ethnotheories that unite the knowledge and beliefs of certain sociocultural groups about the upbringing of children [19, 20, etc.]. The ethnic parenting theories reflect knowledge and representations on a range of positions - from the duration of breastfeeding and the degree of emotional support to developmental milestones and the value of educational activities in a child's life.

For example, it was repeatedly stated that children's education was far more important for Asian parents than for European and American parents which, for example, results in higher rates of educational activities in Chinese families $[21,22]$. It is shown that representatives of a certain cultural community in terms of parental ethnotheories reflect the norms and expectations of the social environment not only in the upbringing of children but also in their perceptions [15]. 
Moreover, the dependence of the gender specificity of parental ethnotheories on the level of socio-economic development is shown (for example, [23]). Studies report culture-mediated maternal and paternal positions regarding "male" and "female" activities [24, 25].

In this study, we focused on the characteristics of parental perceptions of children of primary and secondary school age from Russia and Kyrgyzstan.

The samples of schoolchildren from these two countries are of particular importance for the purposes of this study. First, Russia and Kyrgyzstan represent European and Asian communities, respectively. Second, these countries have different levels of socio-economic development. Thus, according to the Human Development Report, the Russian Federation and Kyrgyzstan are in different groups of countries, according to the Human Development Index (United Nations Development Program, [26]). Russia occupies the 49th place in the rating and is included in the group of countries with a very high level of development, while Kyrgyzstan is on the $120^{\text {th }}$ position and is included in the group with an average level. Third, the Kyrgyz education system is similar to the Russian education system including classes in Russian in a number of schools starting from first grade. Thus, these similarities and differences open the opportunity to analyze the specificity of the perception of the relationship of mothers and fathers in countries that tend to form European and Asian models of family relationships, using common methods of measuring parental attitudes.

\section{Methods}

\subsection{Participants}

The study involved 1962 children of primary and secondary school age from Russia and Kyrgyzstan. The schools in Russia and Kyrgyzstan were selected according to the level of socioeconomic status including characteristics of the teaching staff, education quality and the name of curricula for school disciplines.

The Russian sample included 501 primary school students aged 6.8 to 11.7 years ( $49.9 \%$ boys) and 438 adolescents aged 10.8 to 16.8 years ( $47.1 \%$ boys).

The Kyrgyz sample included 510 primary school students aged 6.6 to 11.8 years ( $48.5 \%$ boys) and 513 adolescents aged 10.3 to 16.8 years ( $46.3 \%$ boys).

All students filled out questionnaires aimed at assessing the factors of perception of maternal and paternal attitude. Written informed consent was obtained from all parents of the children participating in this study. The analysis of the results was carried out on unidentified data.

\subsection{Measure}

Maternal and paternal attitude was assessed with the Russian-language version of the Children's Report of Parental Behavior Inventory (CRPBI) [1, 27].

The Children's Report of Parental Behavior Inventory has a four-factor structure, in which the following scales are distinguished: 'Acceptance', 'Positive involvement', 'Rejection' and 'Hostile detachment' [1]. The scale 'Acceptance' consists of 8 statements (for example, 'Cheers me up when I'm sad'); 'Positive involvement' includes 7 statements (for example, 'Is very interested in what I am learning at school'); 'Rejection' consists of 7 items (for example, 'Almost always complains about what I do' and 'Gets cross and angry about little things I do'); 'Hostile detachment' includes 8 statements (for example, 'Wishes I were a different kind of person').

All participants filled out the questionnaire for both parents.

\section{Results}

\subsection{Descriptive statistics}

Table 1 presents the descriptive statistics for maternal and paternal perception on the Russian and Kyrgyz samples of primary school children.

Table 1. Means and standard deviations (in brackets) for maternal and paternal attitude on the sample of primary school children.

\begin{tabular}{|c|c|c|}
\hline & Russian & Kyrgyz \\
\hline Acceptance & $20.1(2.7)$ & $21.1(2.9)$ \\
& $19.5(3.9)$ & $20.6(3.7)$ \\
\hline Positive & $17.8(2.4)$ & $18.5(2.5)$ \\
Involvement & $17.3(3.0)$ & $18.1(3.3)$ \\
\hline Rejection & $10.1(2.9)$ & $9.7(3.2)$ \\
& $10.0(3.1)$ & $9.6(3.4)$ \\
\hline Hostile & $11.7(3.5)$ & $11.0(3.8)$ \\
Detachment & $11.7(3.6)$ & $11.0(4.0)$ \\
\hline
\end{tabular}

Note: top line - maternal attitude, bottom linepaternal attitude.

According to Table 1, higher average values for positive perceptual factors of both maternal and paternal attitudes (Acceptance and Positive Involvement) were obtained on the Kyrgyz sample of primary school students than on the Russian sample. On average, primary school age children report higher emotional acceptance and positive involvement of mothers than fathers.

For negative factors - Rejection and Hostile Detachment - the indicators of Russian schoolchildren are higher than of their Kyrgyz peers, both for mothers and fathers. Table 1 also shows that both Russian and Kyrgyz children perceive the negative attitude of both parents to the same extent.

Table 2 presents descriptive statistics for paternal and maternal attitude on Russian and Kyrgyz samples of adolescents.

In adolescence, unlike at primary school age, the mean values of both positive and negative factors of the parental attitude practically coincide for the Russian and Kyrgyz samples. For example, the average value of maternal Hostile Detachment for schoolchildren in both samples was 10.7, and Russian and Kyrgyz children 
estimate Positive Involvement at 17.8 and 17.9 points, respectively.

Table 2. Means and standard deviations for paternal and maternal attitude on adolescent sample.

\begin{tabular}{|c|c|c|}
\hline & Russian & Kyrgyz \\
\hline Acceptance & $20.0(3.2)$ & $20.2(3.1)$ \\
& $18.9(4.2)$ & $19.5(3.8)$ \\
\hline Positive & $17.8(2.5)$ & $17.9(2.6)$ \\
Involvement & $16.5(3.4)$ & $17.1(3.1)$ \\
\hline Rejection & $9.6(2.7)$ & $9.7(2.9)$ \\
& $9.5(3.2)$ & $9.0(2.6)$ \\
\hline Hostile & $10.7(2.9)$ & $10.7(3.8)$ \\
Detachment & $11.0(3.7)$ & $10.6(3.2)$ \\
\hline
\end{tabular}

Note: top line - maternal attitude, bottom line paternal attitude.

For positive aspects, maternal attitude for both age groups was rated slightly higher than paternal attitude. For negative aspects, there were multidirectional tendencies for mothers and fathers.

\subsection{ANOVAs}

To study the cross-cultural differences in the perception of a child by parents, a one-way ANOVA was applied. The analysis was carried out separately on two agespecific samples: primary school age children and adolescents. Being a resident of the Russian Federation or the Kyrgyz Republic was used as a categorical factor and the indicators of maternal and paternal attitude were consistently introduced as dependent variables.

Levene's test was used to test the hypothesis of the equality of variances of all distributions of the analyzed variables for the compared samples.

Table 3 summarizes the results of the variance analysis on the perception of parental attitude conducted on both samples. Maternal (top line) and paternal attitude (bottom line) were analyzed: Acceptance (A), Positive involvement (PI), Rejection (R) and Hostile Detachment (HD).

According to Table 3, there are small but statistically significant differences in the perception of parental attitudes among Russian and Kyrgyz schoolchildren. To the greatest extent these differences are manifested on the sample of primary school age. Thus, Kyrgyz children perceive the attitude of mothers and fathers as more emotionally accepting and less rejective than their Russian peers. In particular, among the aspects of children's perception of maternal attitude, significant effects of the country were found for such indicators of family microenvironment as Acceptance, Positive Involvement and Hostile Detachment. At the same time, Kyrgyz children's perceptions of maternal attitude are higher for positive scales (Acceptance and Positive Involvement) and less for Hostile Detachment compared to Russian children. Attention is drawn to the small effect size for these scales - from $1 \%$ to $3 \%$. For the aspects of perception of paternal attitude, similar results were obtained: cross-cultural differences were obtained for all analyzed aspects with effect sizes of $1-2 \%$.

Table 3. Estimation of the effect of the country on indicators of parental attitude.

\begin{tabular}{|c|c|c|c|c|c|}
\hline Sample & Indicator & SS & $\mathbf{F}$ & $p$ & $\eta^{2}$ \\
\hline \multirow{4}{*}{ 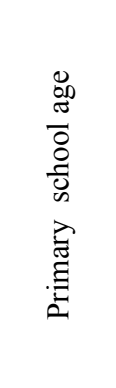 } & A & $\begin{array}{l}275.5 \\
282.9\end{array}$ & $\begin{array}{l}35.1 \\
19.6\end{array}$ & $\begin{array}{l}0.00 \\
0.00\end{array}$ & $\begin{array}{l}0.03 \\
0.02\end{array}$ \\
\hline & PI & $\begin{array}{l}107.2 \\
129.9\end{array}$ & $\begin{array}{l}17.5 \\
13.0\end{array}$ & $\begin{array}{l}0.00 \\
0.00\end{array}$ & $\begin{array}{l}0.02 \\
0.01\end{array}$ \\
\hline & $\mathrm{R}$ & $\begin{array}{l}32.4 \\
46.4\end{array}$ & $\begin{array}{l}3.5 \\
4.4\end{array}$ & $\begin{array}{l}0.07 \\
0.04\end{array}$ & $\begin{array}{l}0.00 \\
0.01\end{array}$ \\
\hline & HD & $\begin{array}{l}126.5 \\
127.9\end{array}$ & $\begin{array}{l}9.3 \\
8.7\end{array}$ & $\begin{array}{l}0.01 \\
0.01\end{array}$ & $\begin{array}{l}0,01 \\
0,01\end{array}$ \\
\hline \multirow{4}{*}{ 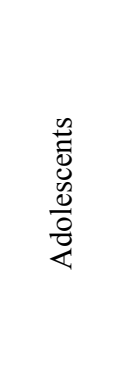 } & A & $\begin{array}{l}16.4 \\
62.9\end{array}$ & $\begin{array}{l}1.7 \\
3.9\end{array}$ & $\begin{array}{l}0.19 \\
0.04\end{array}$ & $\begin{array}{l}0.00 \\
0.01\end{array}$ \\
\hline & PI & $\begin{array}{c}3.4 \\
71.0\end{array}$ & $\begin{array}{l}0.5 \\
6.8\end{array}$ & $\begin{array}{l}0.47 \\
0.01\end{array}$ & $\begin{array}{l}0.00 \\
0.01\end{array}$ \\
\hline & $\mathrm{R}$ & $\begin{array}{c}0.5 \\
41.1\end{array}$ & $\begin{array}{l}0.1 \\
5.0\end{array}$ & $\begin{array}{l}0.80 \\
0.03\end{array}$ & $\begin{array}{l}0.00 \\
0.01\end{array}$ \\
\hline & HD & $\begin{array}{c}0.1 \\
39.8\end{array}$ & $\begin{array}{c}0.01 \\
3.4\end{array}$ & $\begin{array}{l}0.92 \\
0.07\end{array}$ & $\begin{array}{l}0.00 \\
0.00\end{array}$ \\
\hline
\end{tabular}

Note: $S S$ - sum of squares, $F-F$-statistic, $p$ - significance level, $\eta^{2}-$ effect size

On the sample of adolescents, cross-cultural differences were only found in the perception of paternal attitude. In particular, Kyrgyz adolescents perceive paternal attitude as more emotionally supportive and less rejecting than Russian adolescents. It should be noted that the effect sizes are minimal $-1 \%$ of the variance of Acceptance, Positive Involvement and Rejection. Perceptions of maternal attitude did not differ on the samples of Russian and Kyrgyz adolescents.

\section{Discussions}

According to the results of this study, regardless of the conditions of the macroenvironment, primary school age children and adolescents perceive parental attitude more as emotionally accepting and less as rejecting.

At the same time, for the positive aspects of the parental attitude - Acceptance and Positive Involvement - differences in the perception of maternal and paternal attitude were revealed. The children assessed the maternal attitude as more emotionally accepting than the attitude of fathers. In addition, it was found that for children maternal attitude was related to positive involvement in their lives more than paternal attitude.

Similar results were obtained in a comparative study of the educational impact of fathers and mothers on a sample of older preschool children [1,9]. In particular, according to parents' self-reports mothers encouraged the information flow for a child more than fathers meeting the needs of the child and participating in child's 
activities and games [9]. According to the literature, in most cultures the closest parent is a mother who spends more time with the child than their father [7]. In a number of studies this fact is used as an explanatory category in interpreting the exposure model of the family microenvironment influence on test indicators of abilities assuming that the effect size of parents' influence is directly proportional to the time of their communication with the child $[10,28]$.

The study also shows that the negative aspects of parental attitude - Rejection and Hostile Detachment do not differ in terms of perceptions of both maternal and paternal attitudes.

According to the results of this study, features of the macroenvironment affect the perception of parental attitude. At the same time, the macroenvironmental effects are more pronounced in children's perception of paternal attitude.

Indeed, research on child-parent relationships reported stable cross-cultural differences in the degree of emotional acceptance and the nature of parental involvement in child's activities. These differences are associated with the existence of parental ethnotheories suggesting the knowledge and views of parents from a particular cultural group regarding the upbringing and child development $[19,20,29$, etc.]. It was shown, for example, that Chinese parents (both residing in the US and the People's Republic of China) were more involved in the process of educating their children than European parents [21, 22].

It is reported that during the school age child-parent relations and their perception by the growing child are undergoing multiple changes [1]. According to our results, cross-cultural differences in the perception of maternal and paternal attitude are subject to developmental patterns. In particular, cross-cultural differences in the perception of maternal attitude are only manifested at primary school age: Kyrgyz children assess the attitude of mothers as more hostile with positive involvement and less rejection. At the same time, similar cross-cultural differences in the perceptions of paternal attitudes are observed both at primary school age and in adolescence.

It turns out that in less favorable socio-economic conditions of the macroenvironment, the perception of parental attitude is more positive. At first glance, this might seem a paradoxical result. However, it may be explained from the position of a higher level of requirements for the knowledge and skills of the child when entering the school in more favorable conditions of the macroenvironment, in particular, the conditions of education. Thus, Russian children perceive the attitude of their mothers to a lesser extent as accepting and associated with positive involvement at primary school age. According to the data of the Russian longitudinal study, maternal attitude to the child is most influenced by the macroenvironment at older preschool and primary school age, when the child prepares for and / or starts going to school [30]. In particular, an increase in exigence, increased control over the actions of the child and more active participation in child's education were reported.
Thus, the more favorable is the macroenvironment (and, therefore, the higher is the quality of education), the more actively mothers are included in the learning process aimed at increasing the level of knowledge of a preschool child. Such activity of Russian mothers may result in a decrease in child's perception of maternal attitude on the positive aspects of the family microenvironment. On the contrary, in less favorable macro-environment conditions, which imply a lower level of education and lower requirements for children entering schools, maternal attitude is less subject to change. Thus, the features of the macro environment affect the perception of maternal attitude associated with emotional acceptance and positive involvement only at primary school age.

At the same time, this study shows that cross-cultural differences in perceptions of paternal attitudes are manifested at both primary and secondary school age: Kyrgyz children assess paternal attitude more positively than their Russian peers. In particular, Kyrgyz schoolchildren perceive the attitude of their fathers as more emotionally accepting with positive involvement and less emotionally rejecting compared to their Russian peers. This trend might be inversely related to the amount of time a child spent with the father. This fact confirms the analysis of the composition of the families participating in the study and the employment of mothers. In particular, the number of unemployed mothers (and, consequently, overworking fathers) in the sample of Kyrgyz schoolchildren was greater than in the sample of Russian schoolchildren.

Thus, the effects of the influence of the macro environment on the perception of parental attitude are subje to developmental patterns. Features of the macro environment determine the specificity of perception of mothers only at primary school age: Kyrgyz children perceive the attitude of mothers as more emotionally accepting and less rejective than Russian primary school children. Perception of fathers depends on the characteristics of the macro environment throughout the primary and secondary school years: Kyrgyz children assess paternal attitude as more accepting than their Russian peers.

This study was supported by the grant from the Russian Science Foundation [grant RSF №17-78-30028].

\section{References}

1. T.N. Tikhomirova, S.B. Malykh, Cognitive bases of individual differences in academic achievement (Nestor-History, Moscow, 2017)

2. E. Pougnet, L.A. Serbin, D.M. Stack, A.E. Schwartzman, Canadian Journal of Behavioural Science/Revue canadienne des sciences du comportement, 43,173 (2011)

3. C.S.-S. Cheung, E.M. Pomerantz, Child Development, 82, 932 (2011)

4. G.T. Harold, J.J. Aitken, K.H. Shelton, Journal of Child Psychology and Psychiatry, 48, 1223 (2007) 
5. T.N. Tikhomirova, Psychology. Journal of High School of Economic, 4, 133 (2004)

6. C. Kagitcibasi, Journal of Cross-Cultural Psychology, 36, 403 (2005)

7. O.A. Karabanova, Psychology of family relations and the basics of family counseling (Gardariki, Moscow, 2004).

8. R.P. Rohner, R.A. Veneziano, Review of General Psychology, 5, 382 (2001)

9. T.N. Tikhomirova, Voprosy psihologii, 1, 85 (2011)

10. T.N. Tikhomirova, S.B. Malykh, I.A. Lysenkova, A.P. Belova, O.N. Ovcharova, E.D. Gindina, D.A. Gaysina, Theoretical and experimental psychology, 6, 33 (2013)

11. I. Tsaousis, K. Mascha, T. Giovazolias, Child Psychiatry \& Human Development, 43, 238 (2012)

12. J.C. Schwarz, M.L. Barton-Henry, T. Pruzinsky, Child Development, 56, 462 (1985)

13. J. Roksa, D. Potter, Sociology of Education, 84, 299 (2011)

14. A. Lareau, Unequal childhoods: Race, class, and family life (University of California Press, Berkeley, CA, 2003).

15. J.W. Berry, Cross-cultural psychology: Research and applications (Cambridge University Press, Cambridge, UK, 2002)

16. R.K. Chao, Journal of Applied Developmental Psychology, 21, 233 (2000)

17. S.L. Pong, L. Hao, E. Gardner, Social Science Quarterly, 86, 928 (2005)

18. D.Y.F. Ho, The psychology of the Chinese people (Oxford University Press, New York, 2008)

19. S. Harkness, P.O. Zylicz, C.M. Super, B. WellesNyström, M.R. Bermúdez, S. Bonichini, ... \&
C.J. Mavridis, Journal of Family Psychology, 25, 799 (2011)

20. K.H. Rubin, O.B. Chung, Parenting beliefs, behaviors, and parent-child relations: A crosscultural perspective (Psychology Press, New York, 2013)

21. M. Rodic, X. Zhou, T. Tikhomirova, W. Wei, S. Malykh, V. Ismatulina, E. Sabirova, Y. Davidova, M. Tosto, J-P. Lemelin, Y. Kovas, Developmental Science, 18, 165 (2015)

22. X. Zhou, J. Huang, Z. Wang, Z. Zhao, L. Yang, Y. Zhengaheng, Early child development and care, 176, 763 (2006)

23. C. Kagicibasi, Family and human development across cultures: A view from the other side (Erlbaum, Mahwah, 1996)

24. A.H. Eagly, W. Wood, Behavioral and Brain Sciences, 28, 281 (2005)

25. C.L. Martin, O. Kornienko, D.R. Schaefer, L.D. Hanish, R.A. Fabes, P. Goble, Child Development, 84, 921 (2014)

26. United Nations Development Programme, http://hdr.undp.org/sites/default/files/2016_human_ development_report.pdf(2016)

27. S. Schludermann, E. Schludermann, Notes of the CRPBI-30. (University of Manitoba, Winnipeg, Manitoba, Canada, 1988)

28. T.N. Tikhomirova, S.B. Malykh, The European Proceedings of Social \& Behavioural Sciences, 33, 367 (2017)

29. H. Keller, B. Lamm, M. Abels, R. Yovsi, J. Borke, H. Jensen , ... \& Y. Su, Journal of Cross-Cultural Psychology, 37, 155 (2006)

30. I.A. Luzshetskaya, O.N. Pavlova, Parents and children (Kogito-centre, Moscow, 2003) 